\title{
Analysis of Complex Chilled Water Circuits Using Finite Element Based Modeling
}

\author{
Anjana Matarage ${ }^{1}$, Kapila Perera ${ }^{1}$ and Rahula Attalage ${ }^{1}$ \\ ${ }^{1}$ University of Moratuwa, Sri Lanka
}

\begin{abstract}
High rise buildings often require primary - secondary chilled water circuits due to technical limitation of components. Primary-Secondary chilled water systems usually operate inefficiently in part load conditions due to complex thermal characteristics. Such systems tend to consume more pumping energy due to lower temperature drops in secondary circuits. Additionally deficit flow problem may occur due to improper design of the system which result in lack of cooling. A Finite Element Method (FEM) based modeling approach is presented here which can be used for both optimizing and for input of controlling logic of a primary-secondary chilled water circuit. This involves a model containing chilled water flow and frictional head loss. Model was simulated in Matlab software for optimized design parameters and to calculate the optimum flow conditions during operation to achieve optimum performance. Increasing the number of floors in the secondary circuit decreases the efficiency while by increasing the pumping energy and total head loss of the system.
\end{abstract}

Keywords: HVAC, Simulation, FEM

\section{Introduction}

HVAC system is a major energy consuming component of modern buildings, being accountable for 30-60\% of total building energy consumption [1]. Since most of the central air conditioning systems employ chilled water circulation systems, pumping of chilled water is also a significant contributor to energy consumption [2]. With the increase of height of building, static pressure in the chilled water circuit increases. To overcome developing high pressures inside the circuit, designers will cascade the system in to several isolated circuits thermally coupled through heat exchangers. Even though this design strategy reduces the equipment costs, systems face with several challenges during operation. Low temperature differences in chilled water supply and return and high pressure drops in chilled water system are two of the critical issues observed in cascaded chilled water circuits. These issues are result of either below optimum design or improper operation and set points of the system [3]. Several design options are available for chilled water circuits. They include primary only and primary - secondary systems involving fixed and variable pump duty points [5], [6].

Primary secondary chilled water circuits are the main option for high rise buildings. The main limiting factor for the chilled water circuit to be cascaded is the static pressure inside the system. Higher the static pressure inside the circuit, higher the cost of equipment such as chiller, cooling coils etc. Therefore it is preferred minimize the static pressure inside the system. Chiller and other components of the circuit are limited in withstanding pressure. The highest pressure rating for chillers available was found to be 25 bar during the field surveys. But these high pressure rated chillers are also high in cost. Therefore it is preferable to use a low pressure rating chiller and other cooling equipment.

Even an optimally designed system may operate below optimum at a part load condition. This will always be present due to the safety factors applied to the system and operating conditions. Therefore most commonly a chilled water system will operate in part load conditions. Therefore it is required to formulate a system to operate the system optimally during practical operations [12]. Multiple methods are discussed in literature for control strategy of a plant. [7], [8], [1] But the scope of this paper is limited to optimizing the design of the chilled water plant. 


\section{Modeling Approach}

Several methods were evaluated for modeling the chilled water circuit in order to optimize. Main targets was to use an existing software package for the modeling. Watercad, Comsol Multiphysics, Energy Plus and Equest were evaluated for feasibility.These software did not support extracting operating variables and iterative solutions of the system which was required for arriving at the solution. Therefore it was decided to develop a model using basic Finite Element Method (FEM) equations. The FEM model explained below enables to access and manipulate operating parameters of each component of the chilled water circuit.

Typical layout of a primary secondary chilled water system is given in figure 01. This is a general illustration used as baseline to develop the model. It can be expanded to represent a more complex setup. Main aim of the model to include a primary-secondary combined chilled water circuit. Each component of the system is modelled hydraulically to include the head loss characteristics. Once the model was completed, it was simulated to arrive at the minimum pumping energy consumption while maintaining the required cooling conditions at the building. Each of the mode components is converted in to a fluid flow element to finally include in the FEM model. Hydraulic pressure drop in each component is modelled through the equation

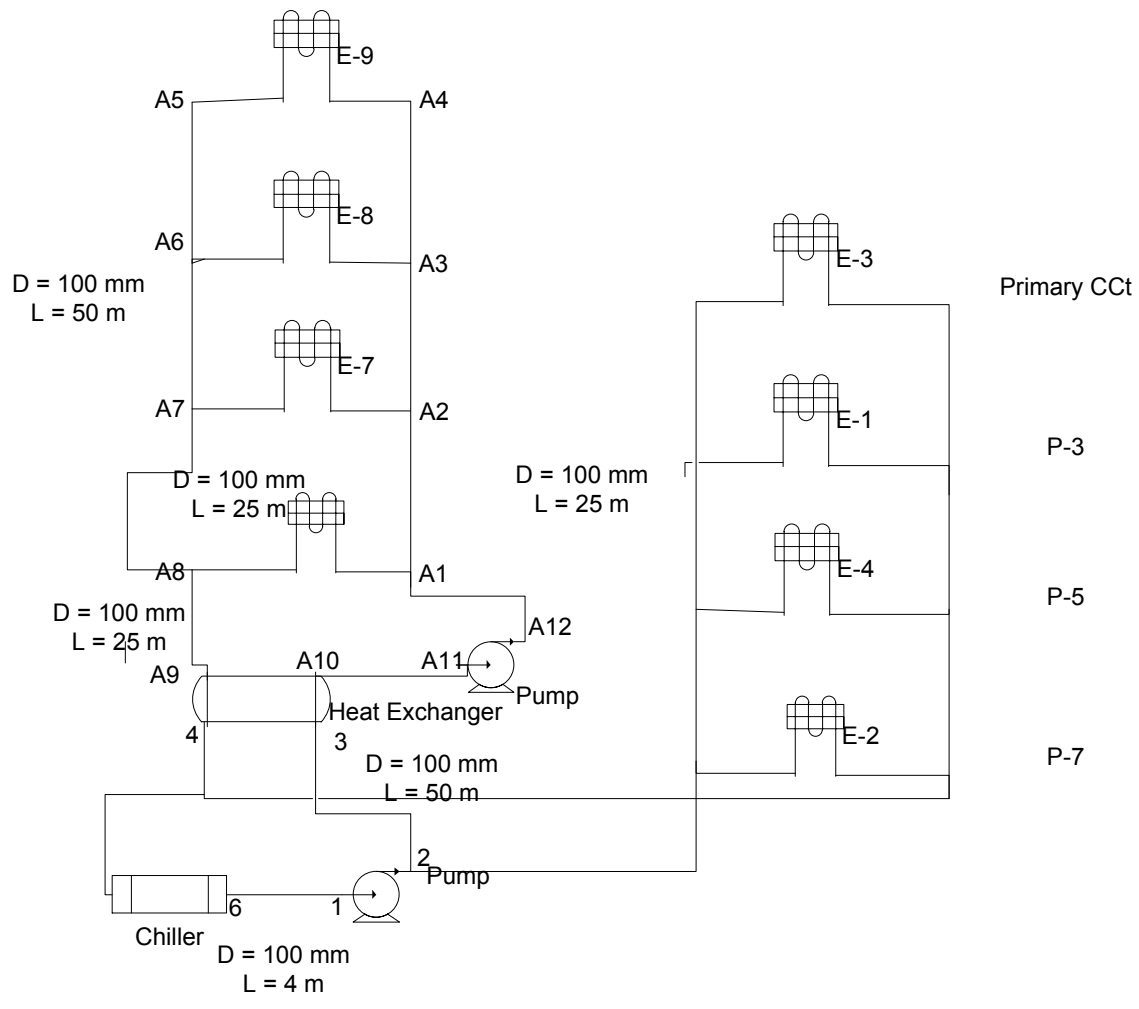

Figure 1: Model Configuration

\subsection{Heat Exchanger}

Heat exchanger discussed here is Tubular Heat Exchanger (THE). Modeling equation (1) given in ashrae handbook is used to calculate the pressure drop through each of primary and secondary circuits [9] where $\Delta \mathrm{P}$ is pressure drop, $\mathrm{Np}$ is number of tubes, $\mathrm{K}_{\mathrm{H}}$,

$$
\Delta \mathrm{P}=\mathrm{Np}\left(\mathrm{K}_{\mathrm{H}}+\mathrm{fL} / \mathrm{D}\right) \rho v^{2} / 2
$$

\subsection{Pipes}

Hazen Williams formula for pipes flowing in full, is used to calculate the head losses in pipe lines [10] as given by equation (2) where $\mathrm{D}$ is pipe diameter, $\mathrm{c}$ is frictional coefficient, $\mathrm{L}$ is length, $\rho$ is density, $\mathrm{g}$ is gravity and $\mathrm{P}$ is pressure. Pipe sections are considered as separate individual elements. 


$$
\mathrm{F}^{1.85}=-\frac{D^{4.87} \times C^{1.8 b}}{10.67 L \rho g} \times(P 3-P 2)
$$

\subsection{Chiller}

Head loss in a chiller is specified by the manufacturer. The value needed to be expressed as a function of flow rate. Since the suppliers provide these details as graphs, a curve fitting technique is used to derive the governing equation. It is observed that a second order equation fits the curve as given in equation (3) below where $\mathrm{F}$ is flow rate and A,B,C are constants. Chiller selected for this model is a 'Carrier' chiller for which the manufacturer's literature is available.

$$
\mathrm{P} 5-\mathrm{P} 6=\mathrm{A} \mathrm{F} \mathrm{F}^{2}+\mathrm{B} \mathrm{F}+\mathrm{C}
$$

A sample equation to represent a chiller head loss:

$$
\mathrm{P}_{2}-\mathrm{P}_{1}=0.2184 \mathrm{~F}^{2}+1.4417 \mathrm{~F}-0.6116
$$

\subsection{Air Cooling Equipment;}

A general chilled water circuit can consist of either or both fan-coil units and air handling units (AHUs). Fan coil units are selected to develop this model. Properties of the fan coil units are taken from the manufacturer's specifications. To allow for different types of cooling equipment, the hydraulic pressure loss is converted in to an equivalent length pipe of the same diameter as secondary circuit.

\section{FEM Model}

Primary and secondary circuits are developed as separate models linked through the heat exchanger. But the hydraulic circuits behave independently. Building block of the model is given in equation 4 [11]. This is the basic model development for a fluid flow element with two nodes. Since the primary circuit contain only series elements, head loss is directly calculated using the equations.

$$
\left\{\begin{array}{l}
f_{1} \\
f_{2}
\end{array}\right\}=\frac{A K_{x x}}{L}\left[\begin{array}{rr}
1 & -1 \\
-1 & 1
\end{array}\right]\left\{\begin{array}{l}
p_{1} \\
p_{2}
\end{array}\right\}
$$

The secondary circuit has multiple parallel connections. Therefore the solution cannot be achieved directly from solving the equations. It is modeled using FEM equations. Each component such as a pipe section or a FCU is considered as two nodes of the final $n \times n$ FEM matrix. Each FCU is assigned with fixed chilled water flow to ensure that the solution model does not suffer from deficit flow problem or low delta $\mathrm{T}$ at higher levels of the buildings.

\subsection{Assembling the Individual Circuit Models}

Each two node element needs to be assembled to arrive at the final FEM model of the circuit. Initially the finite FEM equation was developed in the MATLAB program for selected specifications. The initial matrix contain null values. Then each element is placed in the respective position in the final assembled FEM equation by navigating through the elements of the FEM matrix. This is done through three separate scripts. Main script is used to calculate element flow rates, take user inputs and call other scripts with corresponding values. Two more scripts are used to calculate create and solve primary and secondary circuits separately. Figure 2 shows the program flow for script of calculating primary circuit properties. 


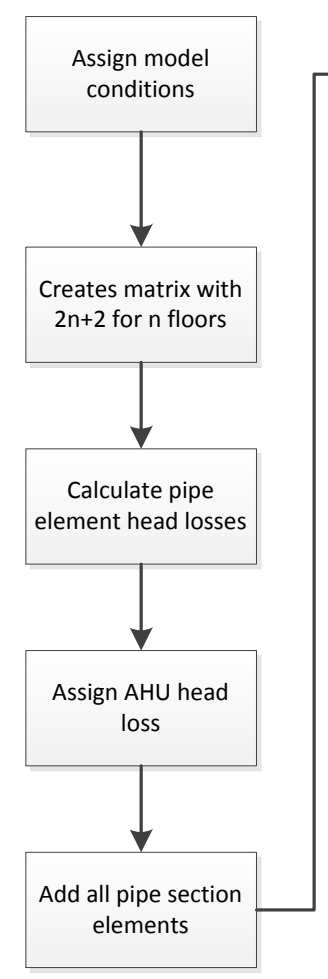

Figure 2: Primary circuit solution flow

\subsection{Simulation}

Simulation is carried out by solving the FEM matrix achieved by assembling all elements of the circuit. The result matrix directly gives the pumping head loss of the system. Head loss is used to calculate the pumping energy required for each of primary and secondary circuits. Simulation conditions were set for a 30 floor building. Model capability is not limited by number of floors. It can be set dynamically according to the application. Simulation was iterated for different combinations of primary and secondary circuit sizes. Each node is simulated at an assigned flow rate. Therefore the flow rates calculated model will ensure correct operating conditions of cooling air cooling equipment.

\section{Results and Analysis}

According to the simulation results, the lowest pumping energy is consumed when the secondary circuit has the minimum number of floors. This is expected since the secondary circuit introduces more losses with heat exchanger and additional piping. Therefore the secondary circuit has to be minimized to improve the energy efficiency of chilled water circuit. But the maximum number of floors that can be included in secondary circuit is limited by the available pressure ratings of chillers and other components. Therefore it is advisable to minimize the number of secondary floors while maintaining the static pressure in the primary circuit within permissible levels. It was observed that pressure is limited to 20 bar in practical applications. Moving the position of cascading heat exchanger upwards by 1 floor will have an approximate reduction of $0.8 \%$ of total pumping head loss under ideal conditions.

Solution of the FEM matrix is not available if the set flow is not achievable at air cooling equipment. Therefore this model can be used to verify if a design will have deficit flow problem during operation. Also the properties of the air cooling equipment elements can be used to determine the controlling conditions of cooling during operating conditions. 


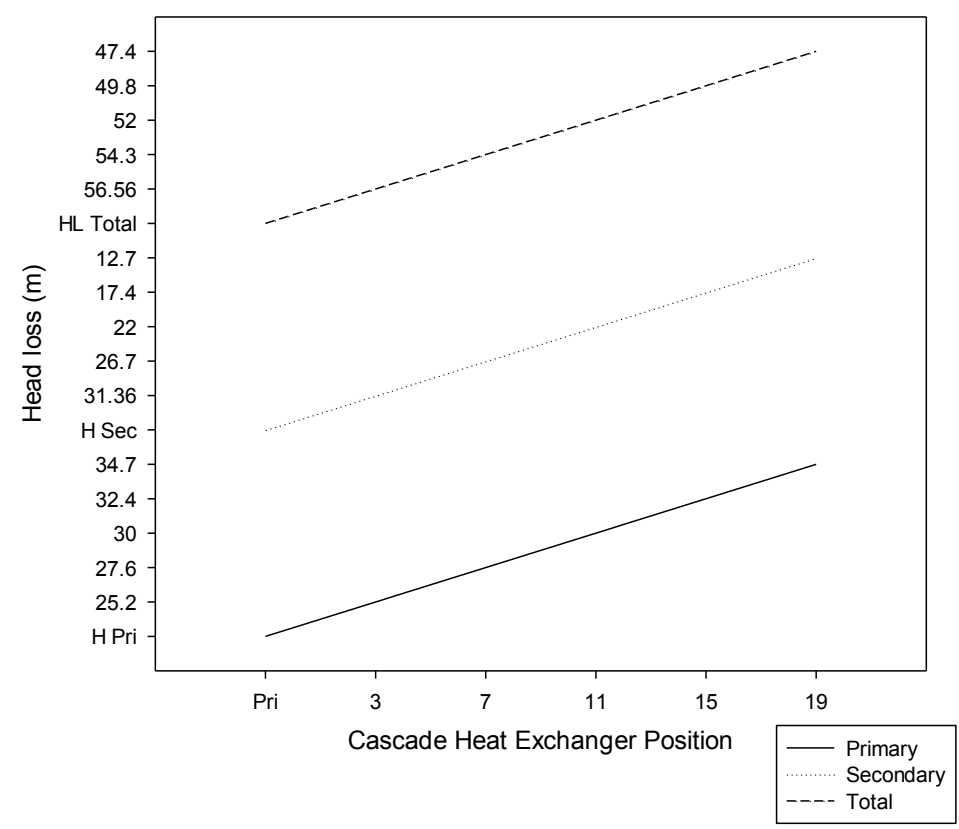

Variation of Flow Rates

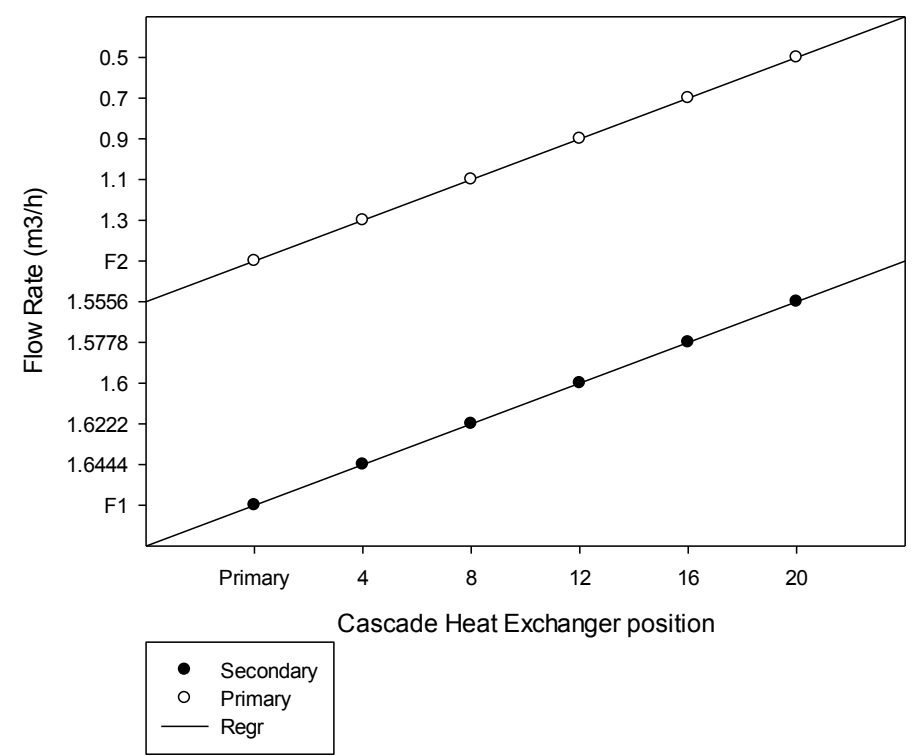

An increase in pipe diameters of primary circuit is suggested to maintain a consistent pressure drop within the system components. In practice, we can see installations which have higher numbers of secondary circuit floors. This is only justified by the high installation cost of components with high pressure rating.

\section{References}

[1] Muzaffar Ali, Vladimir Vukovic, Mukhtar Hussain Sahir, Giuliano Fontanella, Energy analysis of chilled water system configurations using simulation-based optimization, Energy and Buildings, Volume 59, April 2013, Pages 111122, ISSN 0378-7788, http://dx.doi.org/10.1016/j.enbuild.2012.12.011.

[2] J.C. Lam, Energy analysis of commercial buildings in subtropical climates, Building and Environment 35 (2000) 19-26 
[3] Dian-ce Gao, Shengwei Wang, Yongjun Sun, Fu Xiao, Diagnosis of the low temperature difference syndrome in the chilled water system of a super high-rise building: A case study, Applied Energy, Volume 98, October 2012, Pages 597-606, ISSN 0306-2619, http://dx.doi.org/10.1016/j.apenergy.2012.03.057.

[4] Fiorino DP. How to raise chilled water temperature differentials. ASHRAE Trans 2002;108(1):659-65.

[5] Syed A. Tirmizi, P. Gandhidasan, Syed M. Zubair, Performance analysis of a chilled water system with various pumping schemes, Applied Energy, Volume 100, December 2012, Pages 238-248, ISSN 0306-2619, http://dx.doi.org/10.1016/j.apenergy.2012.05.052.

[6] Zhenjun Ma, Shengwei Wang, Enhancing the performance of large primary-secondary chilled water systems by using bypass check valve, Energy, Volume 36, Issue 1, January 2011, Pages 268-276, ISSN 0360-5442, http://dx.doi.org/10.1016/j.energy.2010.10.042.

[7] Zhiqin Zhang, William D. Turner, Qiang Chen, Chen Xu, Song Deng, Optimization of a chilled water plant without storage using a forward plant model, Tenth International Conference for Enhanced Building Operations, Kuwait, October 26-28, 2010

[8] Zhenjun Ma, Shengwei Wang, An optimal control strategy for complex building central chilled water systems for practical and real-time applications, Building and Environment, Volume 44, Issue 6, June 2009, Pages 1188-1198, ISSN 0360-1323, http://dx.doi.org/10.1016/j.buildenv.2008.08.011.

[9] 2008 ashrae handbook Heating, Ventilating, and Air-Conditioning Systems and Equipment (I-P Edition)

[10] Nazith K. Shammas and Lawrence K. Wang, "Water Supply and Wastewater Removal", Fair, Geyer and Okun, Third edition.

[11] Daryl L. Logan, A First Course in the Finite Element Method, Fourth Edition.

[12] John Rauch, Simulation Controlled Large Scale District Energy Chilled Water System Optimization, Pepco Energy Services, Atlantic City, NJ, 2014 